\title{
COVID-19-LOCKDOWN IN WUHAN UND DIE VARIABILITÄT DER HERZRATE - EIN KURZBERICHT ZU EINER PILOTSTUDIE
}

\section{COVID-19 Lockdown in Wuhan and Heart Rate Variability - A Short Report on a Pilot Study}

\section{Zusammenfassung}

Frauen haben offensichtlich eine bessere LockdownBewältigungsstrategie im Hinblick auf den allgemeinen Gesundheitszustand als Männer. Eine transkontinentale Pilotstudie an 50 Personen zur Herzratenvariabilität in Wuhan liefert erste Trendhinweise zu dieser Thematik.

\section{Schlüsselwörter}

COVID-19, SARS-CoV-2, Herzratenvariabilität, Geschlecht, Lockdown, Wuhan

\section{Summary}

Women obviously have better lockdown coping strategy with overall health than men. A transcontinental pilot study in 50 persons on heart rate variability in Wuhan provides initial trend information on this subject.

\section{Keywords}

COVID-19, SARS-CoV-2, heart rate variability, gender, lockdown, Wuhan
$\mathbf{W}$ o, wenn nicht in Wuhan könnten die Auswirkungen eines äußerst strikten Lockdowns auf die Gesundheit der Menschen besser untersucht werden. Unser Forschungszentrum an der Medizinischen Universität in Graz hat seit vielen Jahren eine intensive Kooperation mit der Hubei Universität für Chinesische Medizin in Wuhan und genau dort sind wir u.a. dieser Fragestellung nachgegangen [1]. In einer ersten Pilotstudie konnten wir herausfinden, dass offensichtlich Frauen den weltweit härtesten Lockdown in Bezug auf Veränderungen des allgemeinen Gesundheitszustands, gemessen an derVariabilität des Herzens, besser überwunden haben könnten als Männer.

Die weltweite Verbreitung von COVID-19 hält die Bevölkerung in ständiger Angst. Psychischer Stress und Angstzustände können nicht nur zu psychischen Erkrankungen führen, sondern in schweren Fällen auch den Menschen körperlich und geistig schädigen. Die Herzratenvariabilität (HRV) kann die Aktivität des autonomen Nervensystems widerspiegeln und auch indirekt als objektiver Parameter für einen guten oder schlechten Gesundheitszustand verwendet werden. Ein Vergleich der relevanten HRV-Indikatoren und der mittleren Herzrate
(HR) bei gesunden Menschen vor und nach dem Auftreten von COVID-19 kann daher Hinweise liefern, ob der mentale Stress und die Angst vor COVID-19 und/oder einem Lockdown Auswirkungen auf die psychische Gesundheit haben. Wir haben analysiert, ob es eine Korrelation zwischen der HRV gesunder Menschen vor und nach dem Auftreten von COVID-19 gibt. Zu diesem Zweck wurden insgesamt 50 gesunde Proband $\star^{\star}$ innen (27 Frauen, 23 Männer, 33,4 + 14,9 Jahre) rekrutiert. Der Rekrutierungsort war Wuhan in China. Nach dem Auftreten und dem weltweit ersten und härtesten Lockdown am 23.Jänner 2020 für die Dauer von 76 Tagen in Wuhan wurden 25 gesunde Proband $\star_{i n n e n}$ in die COVID-19-Gruppe (August 2020 bis Januar 2021) integriert.Vor Beginn des Lockdowns (2019) wurden bis Dezember 2019 ebenfalls 25 gesunde Proband $\star_{i n n e n}$ retrospektiv als Kontrollgruppe rekrutiert (Klinische Studienregistrierung: ID NCT02924831). Beide Gruppen erhielten HRV-Tests in Wuhan mit einem Equipment vom Forschungszentrum für Traditionelle Chinesische Medizin der Medizinischen Universität Graz und die HRV-Analysen wurden ebenfalls in Graz durchgeführt. Unter Verwendung der SPSS24.0- 


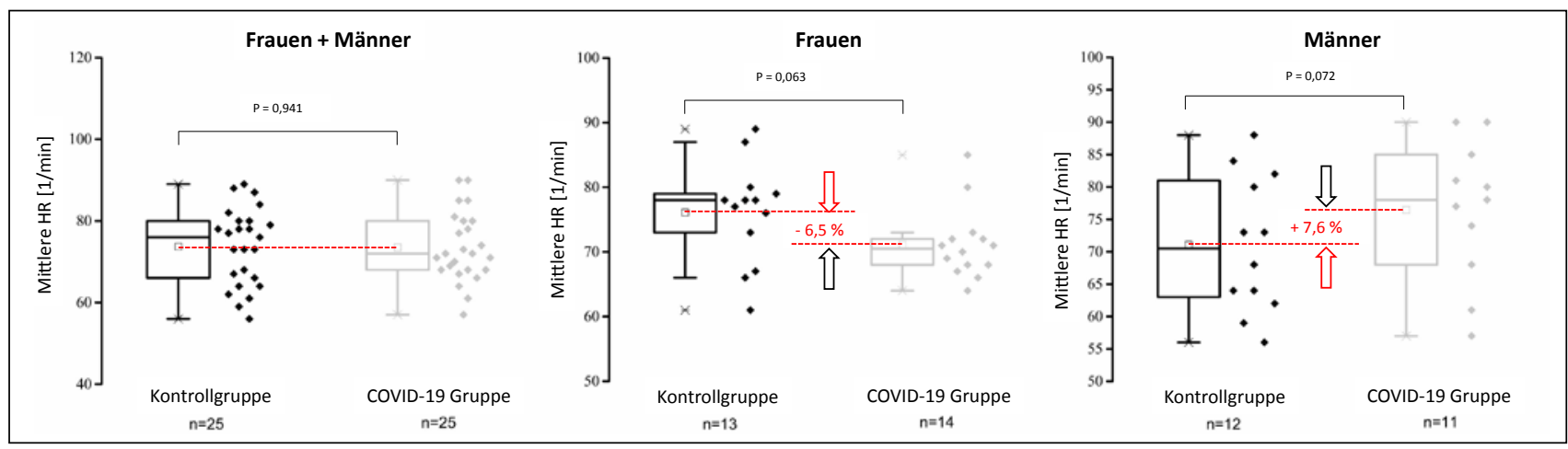

Abb. 1: Box-Plot-Darstellungen der mittleren Herzrate (HR) der Kontroll- und COVID-19-Gruppen

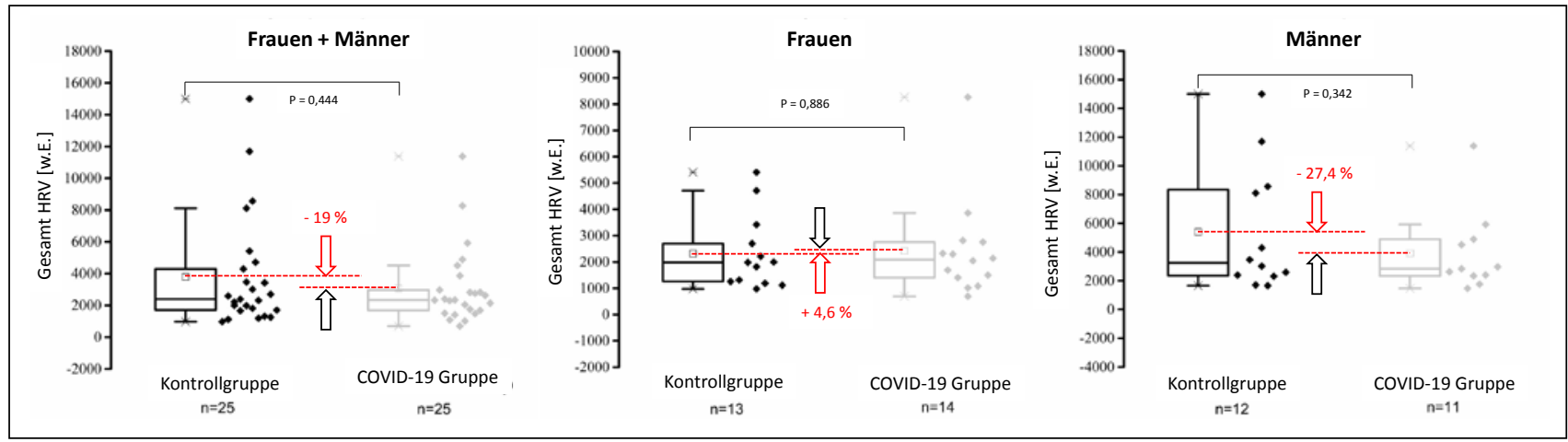

Abb. 2: Box-Plot-Darstellungen der Gesamt-Herzratenvariabilität (HRV) der Kontroll- und COVID-19-Gruppen

Software wurde ein gepaarter Probentest für die Epidemiegruppe und die Kontrollgruppe durchgeführt. Das Signifikanzniveau war mit $\mathrm{p}<0,05$ festgelegt. Die möglichen Änderungen der HRV-Indizes wurden vor und nach dem ersten Lockdown während der COVID-19-Krise verglichen, um mögliche Alterationen des mentalen Stresses und der Angst derVersuchspersonen davor und danach zu erfassen. Zunächst gab es keinen Unterschied bei den HRV-bezogenen Indizes (Gesamt-HRV, SDNN, r-MSSD, VLF, LF, HF, log [LF/HF]) zwischen der COVID-19-Lockdown-Gruppe und der Kontrollgruppe (alle p > 0,05). Der psychische Stress und die Angst vor einem COVID19-Lockdown hatten insgesamt keinen mittelfristigen Einfluss auf die HR und die HRV gesunder Menschen in Wuhan in China. Führte man allerdings in den von der Fallzahl sehr kleinen Gruppen der Pilotstudie eine geschlechtsspezifische Auswertung durch, so konnten wir feststellen, dass offensichtlich Männer imVergleich zu Frauen nach dem Lockdown eine reduzierte Gesamtvariabilität und eine leicht erhöhte mittlere HR aufwiesen (vgl. Abb. 1 und 2).

Diese ersten Ergebnisse des gemeinsamen Forschungsprojekts werden im Mai dieses Jahres auf einer Public Health Konferenz in China vorgestellt.
Die HRV ist ein Parameter, der eine Quantifizierung der Neuromodulation des Herzens ermöglicht. Sie wird in der Forschung und der Praxis nicht nur in der westlichen, sondern auch in der evidenzbasierten traditionellen Medizin immer häufiger eingesetzt. Heute werden innovative Forschungsergebnisse, einschließlich der neuesten Aufzeichnungstechnologie und Techniken der künstlichen Intelligenz, zur Datenerfassung und Datenanalyse der HRV auch in der Akupunktur verwendet.

Die unterschiedlichen Einflüsse auf die HRV sind noch nicht vollständig geklärt, aber es ist bekannt, dass es intraindividuelle und interindividuelle Varianzen gibt und dass die HRV vom Alter abhängt. Abgesehen davon sind zirkadiane Alterationen (Schlaf-Wach-Zyklus), körperliche Verfassung sowie geistige und körperliche Anstrengung wichtige Einflussfaktoren. Die HRV kann auch durch verschiedene Erkrankungen wie altersbedingte und sogenannte Lebensstilkrankheiten, z.B. diabetische Neuropathie, Nierenversagen, essentielle Hypertonie, Herzerkrankungen, Erkrankungen der Herzkranzgefäße oder intrakranielle Läsionen, beeinflusst werden.

Die Erforschung der HRV konzentriert sich am TCM Forschungszentrum der Medizinischen Universität Graz in den letzten Jahren auf neueste innovative Aspekte in Bezug 
auf Komplementärmedizin. Forschungen in Kombination mit verschiedenen Arten von Akupunktur (manuelle Nadelakupunktur, Laserakupunktur, Elektroakupunktur) wurden an mehreren hunderten Patient ${ }^{\star}$ innen, gesunden Freiwilligen und Tieren akquiriert und ausgewertet und der „Scientific Community“ zurVerfügung gestellt (Abb.3) [2].

In China werden Elektrokardiogramme (EKG) registriert und die Daten unmittelbar nach der Erfassung (beispielsweise Akupunkturbehandlung) über das Internet an die Medizinische Universität Graz übertragen. In Graz wird die Analyse der EKG durchgeführt. Die Akupunkteur`innen in China (Harbin, Peking, Wuhan, etc.) werden sofort über die Ergebnisse der Analyse informiert und der Erfolg der Therapie oder das Ergebnis der Studie kann objektiv nachgewiesen werden. Somit stellt diese Art der Teleakupunktur eine mehrfache Brückenfunktion etwa zwischen östlicher und westlicher Medizin, aber auch zwischen Wissenschaft und Praxis dar. Dies kann wesentlich dazu beitragen, redundante Forschungsstudien $\mathrm{zu}$ unterlassen und das diagnostische und therapeutische Verfahren zu vereinfachen, wodurch nicht nur Kosten gesenkt werden, sondern auch Zeit gespart wird.

HRV kann mittlerweile als zuverlässiger Indikator für den Gesundheitszustand verwendet werden. Es konnte gezeigt werden, dass man bei speziellen Syndromen wie Müdigkeit und Stress einem negativen Verlauf mit verschiedenen Präventionsmethoden wie Akupunktur entgegenwirken kann. Dies wurde in Untersuchungen an Patienten mit Burn-out-Syndrom gezeigt, die in transkontinentalen Teleakupunkturstudien zwischen China und Graz durchgefuihrt wurden [3]. Für weitere Forschungsvorhaben während und nach der COVID-19-Pandemie
Abb. 3: Herzratenvariabilität und Akupunktur. Sino-Österreichische Forschungsprojekte zur Telemedizin (EPU 36/2019, EPU $35 / 2019)$

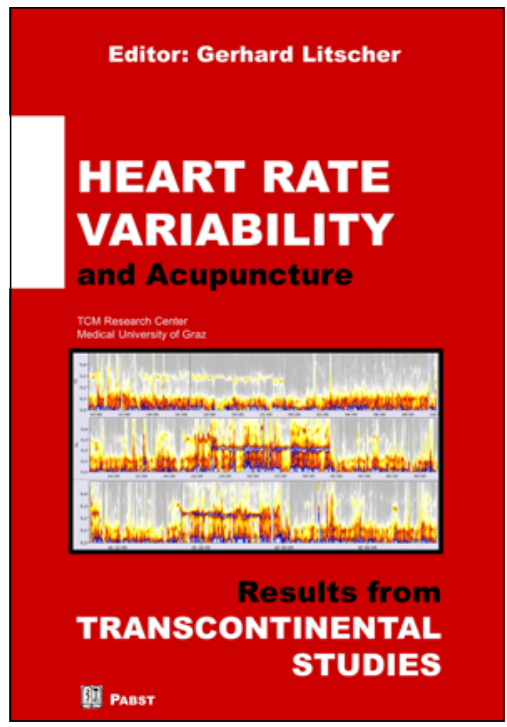

wird es auch sehr interessant sein, einzelne Gruppen mit quantitativ bewertetem Wohlbefinden sowie insbesondere Kinder und Jugendliche zu untersuchen. Quantifizierbare Vergleiche mit Daten von Europäer ${ }^{\star}$ innen [4], die Stress und Angst sowie HRV vor, während und nach einem Lockdown widerspiegeln, wären ebenfalls wünschenswert.

\section{Danksagung}

Der Autor dieses Reports dankt Herrn Li Tong und Frau Prof. Liang Fengxia für die gemeinsame Erarbeitung der vorläufigen Ergebnisse und für die organisatorische und wissenschaftliche Unterstützung in Wuhan. Die Eurasia Pacific Uninet Projekte EPU 35 und 36/2019 (Projektleiter G. Litscher) wurden am 1.4.2020 in Österreich genehmigt.

\section{Literatur}

[1] LiT, Liang FX, Litscher G:The general impact of mental stress and anxiety of COVID-19 on heart rate variability in healthy people in Wuhan, The 5th International Conference on $\mathrm{Pu}$ blic Health and Medical Science, Wuhan, Hubei Province, China, 29-31 May, 2021.

[2] Litscher G (ed.): Heart rate variability and acupuncture. Lengerich: Pabst Science Publischers; 2016.

[3] Shu Q, Wang H, Litscher D, Wu S, Chen L, Gaischek I, Wang L, He W, Zhou H, Litscher G, Liang F: Acupuncture and moxibustion have different effects on fatigue by regulating the autonomic nervous system: a pilot controlled clinical trial. Sci Rep 2016; 25: 37846.

[4] Bourdillon N,Yazdani S, Schmitt L, Millet GP: Effects of COVID-19 lockdown on heart rate variability. PLoS One 2020; 15: $\mathrm{e} 0242303$.

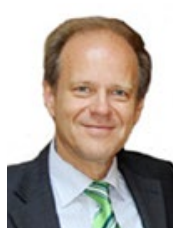

Univ.-Prof. Prof. mult. DI DDr. Gerhard Litscher

Leiter der Forschungseinheit für Biomedizinische Technik in Anästhesie und Intensivmedizin, der Forschungseinheit für Komplementäre und Integrative Lasermedizin

Chairman des TCM Forschungszentrums Graz, Medizinische Universität Graz

Auenbruggerplatz 39, EG19, 8036 Graz, Österreich, E-Mail: gerhard.litscher@medunigraz.at 